\title{
Impact of Sustained Virological Response on Metabolic Profile and Kidney Function in Cured HCV Liver Transplant Recipients
}

\author{
Speranta lacob ${ }^{1,2^{*}}$, Mirela Onica ${ }^{1}$, Razvan lacob ${ }^{1,2}$, Cristian Gheorghe $^{1,2}$, Susanne Beckebaum ${ }^{3}$, \\ Vito Cicinnati ${ }^{3}$, Irinel Popescu ${ }^{4}$, Liana Gheorghe ${ }^{1,2}$
}

\author{
*Corresponding author: \\ Speranta lacob, MD, PhD \\ Center for Digestive Diseases \\ and Liver Transplantation \\ Fundeni Clinical Institute, Bucharest \\ "Carol Davila" University of Medicine \\ and Pharmacy, Bucharest, Romania \\ E-mail:msiacob@gmail.com
}

${ }^{1}$ Center for Digestive Diseases and Liver Transplantation, Fundeni Clinical Institute, Bucharest, Romania

${ }^{2}$ "Carol Davila" University of Medicine and Pharmacy, Bucharest, Romania

${ }^{3}$ University Hospital Muenster, Department of Medicine B, Gastroenterology and Hepatology, Muenster, Germany

4"Dan Setlacec" Center of General Surgery and Liver Transplantation, Fundeni Clinical Institute, Bucharest, Romania

\section{ABSTRACT}

LT - liver transplantation;

HCV - hepatitis C virus;

DAAs - direct-acting antiviral agents;

SVR - sustained virological response;

HCC - hepatocellular carcinoma;

RBV - ribavirin;

LSM - liver stiffness measurement;

TE - transient elastography;

BMI - body mass index;

APRI - AST to Platelet ratio index;

FIB-4 - Fibrosis-4;

eGFR - estimated glomerular filtration rate;

MDRD - Modification of Diet in Renal Disease;

MetS Z score - Metabolic syndrome severity score;

HDL - high-density lipoprotein;

TG - triglycerides;

FRS - Framingham risk score

LDL - low-density lipoprotein;

CNI - calcineurin inhibitor;

CKD - chronic kidney disease.

Received: 20.03.2021

Accepted: 27.05.2021
Background: Long-term morbidity and mortality following liver transplantation (LT) is influenced by HCV-related extrahepatic complications and effects of immunosuppressive therapy.

Aim: To investigate if sustained virological response (SVR) after therapy with direct acting antivirals (DAA) in recipients with post-transplant recurrent hepatitis $C$ can influence metabolic factors and renal function.

Methods: Metabolic profile, cardiovascular risk scores, non-invasive evaluation of fibrosis, renal function was assessed in 89 HCV LT recipients at SVR and 24 months after cure.

Results: Liver stiffness measurement evaluated by transient elastography, APRI, FIB-4 and NAFLD fibrosis scores decreased significantly between baseline, SVR and 24 months after SVR. In contrast, BARD score increased significantly $(p=0.001)$. Steatosis grade 3 was significantly encountered in a higher percentage at 24 months after SVR compared to baseline $(77.5 \%$ vs $22.5 \%, p<0.0001)$. The metabolic and cardiovascular risk profile (MetS and Framingham scores), respectively, remained stable during the timeline. All liver function tests such as alanine and aspartate aminotransferase, gamma glutamyl transferase, total bilirubin improved between initiation of antivirals, SVR and 24 months after SVR; platelets increased significantly $(p<0.0001$ for each variable). The renal function evaluated by creatinine serum level $(p=0.03)$ and estimated glomerular filtration rate $(p=0.02)$ was significantly deteriorated over time.

Conclusions: Eradication of recurrent HCV infection has a clear benefit for liver-related complications, but has no impact on HCV extrahepatic manifestations. Prospective studies with non-HCV cohorts are required to compare the impact of immunosuppression on metabolic and renal complications.

Key words: HCV cure, metabolic complications, cardiovascular profile, liver transplantation

\section{INTRODUCTION}

Nowadays, 20-years patient and graft survival rates after adult liver trans- 
plantation (LT) are over 50\% (1-3). The long term morbidity and mortality after LT is now determined by the presence of complications such as cardiovascular disease, diabetes, metabolic syndrome, renal failure (4). Hepatitis $\mathrm{C}$ virus ( $\mathrm{HCV}$ ) is also involved in the occurrence of these extrahepatic manifestations in addition to effects of immunosuppressive therapy. However, in the study of Dopazo et al. (5) the post-LT 20-years survival rates were only $20 \%$, main reason being the high proportion of patients transplanted for $\mathrm{HCV}$ with recurrence and death following LT.

However, the recent progress of direct-acting antiviral agents (DAAs) associated with very high efficacy rates has also significantly changed the outcome of the HCV transplant population (6). Long-term follow-up studies suggest that HCV cured patients after antiviral therapy achieve multiple benefits: regression of fibrosis, declining risk of evolution to decompensation of liver cirrhosis, decreasing the need for LT and mortality (7).

A recent review demonstrated that $\mathrm{HCV}$ infection is positively correlated with insulin resistance, liver steatosis, metabolic syndrome, type II diabetes mellitus, atherosclerosis and lipid rebound after DAA successful therapy (8). HCV infection has been confirmed to be associated with metabolic syndrome which may worsen the progression of HCV-related liver disease. In the DAA era, the impact of viral clearance on metabolic syndrome and associated diseases requires more exploration, especially in the LT setting.

Two studies published in 2018, revealed that cure of HCV infection by DAA in LT recipients has favourable influence on glucose metabolism, reverses the hypolipidemic effect of HCV and significantly lowers the risk of worsening of renal function $(9,10)$. Fortunately, after the introduction of DAA in the management of our LT $\mathrm{HCV}$ recipients, all obtained sustained virological response (SVR) despite being treated with advanced liver fibrosis (>35\%) (11).

The aim of our study was to explore if SVR influences positively metabolic factors, cardiovascular risk scores, fibrosis scores and renal function.

\section{METHODS}

\section{Patient's selection}

Eighty nine adult patients transplanted for HCVrelated decompensated cirrhosis with or without hepatocellular carcinoma ( $\mathrm{HCC}$ ) at the Centre of Digestive Diseases and Liver Transplantation, Fundeni Clinical Institute and treated with DAAs between December 2015 and December 2017 were included in this observational study. Patients received ombitasvir/ paritaprevir/ritonavir + dasabuvir plus ribavirin (RBV) or ledipasvir/sofosbuvir plus RBV according to the therapeutic guidelines of the Protocol Expert Committee of Ministry of Health and National Health Inssurance Agency. All our patients obtained SVR. All patients had at least a liver biopsy performed during follow-up after LT confirming the diagnosis of recurrent HCV chronic hepatitis. Plasma HCV RNA levels were measured using the Roche TaqMan real-time reverse transcriptase-PCR assay version 2.0 at baseline, end of treatment and SVR (lower limits of quantification and detection of 15 $\mathrm{IU} / \mathrm{mL}$ ). All patients underwent genotyping for $\mathrm{HCV}$ and all proved to be genotype $1 \mathrm{~b}$.

The following parameters were evaluated before initiation of interferon-free regimen, at SVR and 24 months after SVR: metabolic profile (weight/body mass index (BMI), serum cholesterol/LDL/HDL/triglycerides, glucose, steatosis evaluated by ultrasound and CAPFibroscan ${ }^{\circledR}$ ), cardiovascular risk scores (MetS and Framingham scores) $(12,13)$, non-invasive evaluation of fibrosis (AST to Platelet Ration Index - APRI, Fibrosis-4 FIB-4, NAFLD fibrosis score, BARD score, LSM by Fibroscan?]), renal function (serum creatinine, estimated glomerular filtration rate (eGFR) calculated by the Modification of Diet in Renal Disease (MDRD) 6).

The MetS Z score (Metabolic syndrome severity score) includes age, sex, race and ethnicity, height and weight, waist circumference $(\mathrm{cm})$, systolic blood pressure, high-density lipoprotein (HDL) cholesterol $(\mathrm{mg} / \mathrm{dL})$, triglycerides (TG) $(\mathrm{mg} / \mathrm{dL})$, glucose $(\mathrm{mg} / \mathrm{dL})$ and can be easily calculated online (http://mets.healthoutcomes-policy.ufl.edu/calculator/). Each 1 standard deviation increase in score confers an additional increase in odds of 3.4 for future cardiovascular disease and 7.3 for future type 2 diabetes mellitus (14). The risk of future cardiovascular events was estimated as the 10-year probability of cardiovascular disease using the Framingham risk score (FRS) that includes gender, age, HDL-cholesterol, low-density lipoprotein (LDL) - cholesterol, the presence of diabetes mellitus, systolic blood pressure, diastolic blood pressure and smoking status (13).

All patients had interferon-free therapy finalization duration since more than 3 months and were on stable immunosuppression prior to the initiation of antiviral therapy. Stable immunosuppression was defined as steroid cessation for more than 3 months and without any antirejection medication change for more than 3 months. Our transplant centre uses a steroid-free immunosuppression protocol that consists of induction immunosuppression with basiliximab and delayed 
initiation of calcineurin inhibitor (CNI). Mycophenolate mofetil is used during the first 3 months after LT and, on the long term, for nephroprotection in association with $\mathrm{CNI}$ level minimization. Sirolimus is used after month 3 , usually in patients with HCC.

Exclusion criteria were:less than 3 months after LT without stable immunosuppression drugs, confirmation of recurrent $\mathrm{HCC}$ after $\mathrm{LT}$, patients treated and cured from HCV before LT and incomplete antiviral treatment post-transplant, patients on renal replacement therapy after LT.

The influence of HCV treatment on renal function in LT recipients was determined by the variation in serum creatinine levels and eGFR calculated by MDRD-6 between baseline, SVR and beyond 24 posttreatment. Renal dysfunction was defined as eGFR $<60 \mathrm{~mL} / \mathrm{min} /$ $1.73 \mathrm{~m}^{2}$. The other definitions used in the present study were: arterial hypertension - blood pressure over 140/ $90 \mathrm{mmHg}$ at two following visits; diabetes mellitus fasting plasma glucose $>126 \mathrm{mg} / \mathrm{dL}$ at two following visits according to the World Health Organization; dyslipidemia - hypercholesterolemia $>220 \mathrm{mg} / \mathrm{dL}$ and/ or hypertriglyceridemia $>200 \mathrm{mg} / \mathrm{dL}$ at two following visits. Metabolic syndrome was present if three or more of the following five criteria are met: waist circumference over $102 \mathrm{~cm}$ (men) or $88 \mathrm{~cm}$ (women), blood pressure over $130 / 85 \mathrm{mmHg}$, fasting TG level over $150 \mathrm{mg} / \mathrm{dl}$, fasting HDL cholesterol level less than $40 \mathrm{mg} / \mathrm{dl}$ (men) or $50 \mathrm{mg} / \mathrm{dl}$ (women) and fasting blood glucose over $100 \mathrm{mg} / \mathrm{dl}$ (14).

\section{Statistical analysis}

Continuous variables were reported as a mean \pm standard deviation, while categorical variables were reported as frequencies and percentages. Comparisons were made by chi-square-test or McNemar test for qualitative variables; Mann-Whitney-U and Wilcoxon tests were used to compare continuous data. Friedman test (ANOVA) was performed for global comparison of quantitative variables at baseline, SVR and 24 months after achieving SVR. Pearson correlation coefficient was used to investigate the linear correlation between quantitative variables. All statistical tests were two-sided and a level of $P<0.05$ was used to indicate statistical significance. All statistical analyses were carried out using NCSS 11.0 (LLC, Kaysville, Utah, USA).

\section{RESULTS}

Eighty nine HCV LT recipients treated with DAA and SVR, followed up for 24 months after achieving SVR were included. A total of 76 (83.39\%) patients received ombitasvir/paritaprevir/ritonavir + dasabuvir plus RBV and 13 (16.61\%) received sofosbuvir/ledipasvir +/- RBV. The mean age at therapy initiation was $55.7 \pm 7.8$ years and $51.69 \%$ were males. One third $(32.58 \%)$ of LT recipients had F3-F4 stage at baseline and 23 (25.84\%) patients received previous interferon therapy without response. Median time since LT to DAA therapy initiation was 28 months (range 3.5-177.8 months). 48.3\% of patients had less than 2 years since LT when they started DAA. Transplant indications were HCV decompensated cirrhosis in $74.16 \%$ of patients and HCC in $25.84 \%$. Type of immunosuppression at DAA initiation was tacrolimus $(77.27 \%)$, cyclosporine $(14.77 \%)$ and sirolimus (7.96\%). None of the patients received corticosteroids during antiviral therapy or in the follow up period.

Baseline metabolic comorbidities were: arterial hypertension necessitating therapy in $39.08 \%$ of patients, diabetes mellitus (32.58\%), dyslipidemia (19.1\%) and eGFR < $60 \mathrm{ml} / \mathrm{min}(21.3 \%)$.

\section{Evolution of fibrosis stage, liver and renal function and metabolic profile between baseline, SVR and 24 months after SVR}

The evolution of noninvasive fibrosis scores and LSM between the three time points is shown in table 1. LSM evaluated by TE, APRI, FIB-4 as well as NAFLD fibrosis scores decreased significantly between baseline, SVR and 24 months after SVR. In contrast, BARD score increased significantly between initiation of antiviral therapy, SVR and after 24 months of follow up.

Steatosis grade evaluated by Fibroscan ${ }^{\circledR}$ with CAP did not change during the three analyzed time points (median value of CAP score 255.5 at beginning of DAA, 278.3 at SVR, $259.1 \mathrm{~dB} / \mathrm{m}$ at 24 months after SVR, $\mathrm{p}=0.82$ ). However, steatosis grade 3 evaluated by both US and CAP score ( $>290 \mathrm{~dB} / \mathrm{m})$ was significantly encountered in a higher percentage at 24 months after SVR compared to the time of DAA was initiated $(77.5 \%$ vs $22.5 \%, p<0.0001)$.

All liver function tests such as alanine and aspartate aminotransferase, gamma glutamyl transferase, total bilirubin had a significant decrease between initiation of antivirals, SVR and 24 months after SVR; platelets increased significantly ( $p<0.0001$ for each variable). The metabolic profile changes over time since beginning of DAA are shown in table 2. However, the cardiovascular risk profile remained stable during the timeline (table 2).

The renal function evaluated by creatinine serum level and eGFR calculated by MDRD6 was significantly 
Table 1 - Noninvasive fibrosis evaluation before antiviral therapy initiation, at SVR and after 24 months of follow up

\begin{tabular}{lcccc}
\hline Variable & Baseline & SVR & $\begin{array}{c}\text { 24 months } \\
\text { after SVR }\end{array}$ & P value \\
\hline Liver stiffness $(\mathrm{kPa})$ & 9.2 & 6.5 & 6.7 & 0.0001 \\
\hline APRI & 1.34 & 0.44 & 0.39 & $<0.0001$ \\
\hline FIB-4 & 2.60 & 1.68 & 1.48 & $<0.0001$ \\
\hline BARD score & 1.76 & 2.19 & 2.03 & 0.001 \\
\hline NAFLD Fibrosis score & 2.44 & 2.12 & 1.94 & 0.02 \\
\hline
\end{tabular}

deteriorated over time since DAA start until 24 months following SVR (fig. 1 and 2). Age at the beginning of antiviral therapy was negatively correlated with the baseline eGFR ( $r=-0.30, p=0.007)$.

There was a significant decrease of eGFR calculated by MDRD6 between baseline and 24 months after SVR in both groups of patients: with $(79.2 \pm 22.8$ vs $69.1 \pm$ $24.1 \mathrm{ml} / \mathrm{min}, \mathrm{p}=0.005$ ) or without diabetes mellitus (78.9 \pm 28.5 vs $69.9 \pm 25.3 \mathrm{ml} / \mathrm{min}, \mathrm{p}=0.006$ ). There was a significant increase (with $19.1 \%, p=0.0002$ ) in the proportion of patients with eGFR $<60 \mathrm{ml} / \mathrm{min}$ at the end of the follow-up period.

There is no statistically significant difference regarding all analyzed variables at the end of the follow-up period in between the patients receiving different types of immunosuppression (tacrolimus versus cyclosporine versus sirolimus). There is also no significant correlation between time since LT and different variables analyzed at the end of the follow-up period.

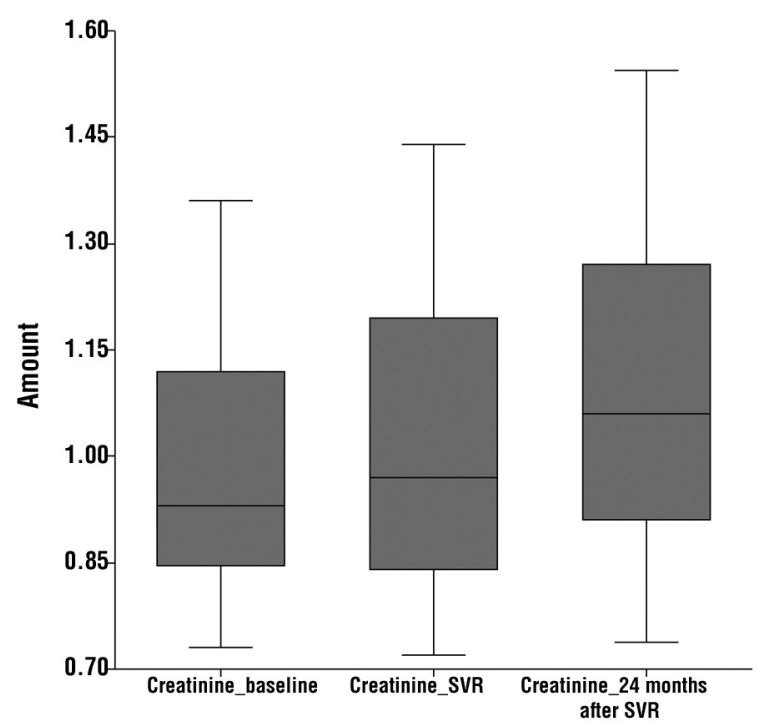

Figure 1 - Serum creatinine $(\mathrm{mg} / \mathrm{dL})$ at baseline, SVR and 24 months after antiviral therapy $(p=0.03)$
Table 2 - Metabolic profile and cardiovascular risk change over time after SVR

\begin{tabular}{lcccc}
\hline Variable & Baseline & SVR & $\begin{array}{c}\text { 24 months } \\
\text { after SVR }\end{array}$ & P value \\
\hline Weight (kg) & 78 & 80 & 84 & $<0.0001$ \\
\hdashline BMI (kg/mp) & 26.3 & 26.9 & 27.3 & $<0.0001$ \\
\hline $\begin{array}{l}\text { Total cholesterol } \\
\text { (normal value 0-200mg/dL) }\end{array}$ & 154 & 174 & 185 & $<0.0001$ \\
\hline $\begin{array}{l}\text { LDL-cholesterol } \\
\text { (normal value 0-130 mg/dL) }\end{array}$ & 95.2 & 97.6 & 110 & 0.23 \\
$\begin{array}{l}\text { HDL- cholesterol } \\
\text { (normal value 5-115 mg/dL) }\end{array}$ & 45.6 & 42.1 & 48.3 & 0.11 \\
\hline $\begin{array}{l}\text { Tryglicerides } \\
\text { (normal value 40-180 mg/dL) }\end{array}$ & 119.5 & 120 & 109.5 & 0.34 \\
\hdashline $\begin{array}{l}\text { Serum glucose } \\
\text { (normal value 74-106 mg/dL) }\end{array}$ & 110.3 & 117 & 126.1 & 0.0009 \\
\hline $\begin{array}{l}\text { MetS score } \\
\text { Framingham score }\end{array}$ & 0.68 & 0.56 & 0.54 & 0.31 \\
\hline
\end{tabular}

\section{DISCUSSION}

DAAs have revolutionized HCV antiviral treatment with very high eradication rates and exceptional safety compared to interferon-based regimens. There are several previous studies $(15,16)$ reporting histological and clinical improvement, associated with rises in patient and graft survival rates after SVR in LT recipients. However, long-term effects of HCV cure after interferon-free therapy on the metabolic condition of patients have been sparsely investigated.

The metabolic syndrome is characterised by obesity, arterial hypertension, high triglycerides, low high-

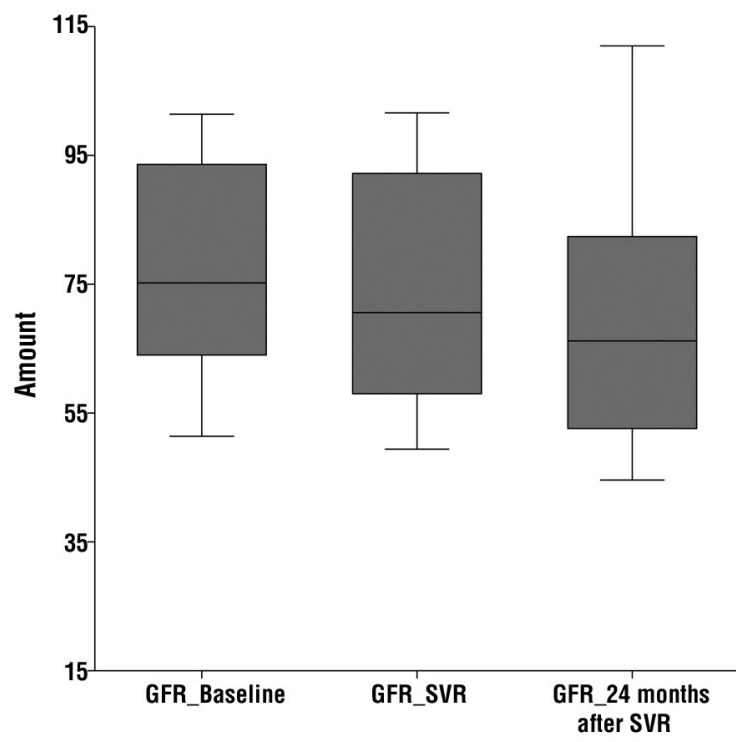

Figure 2 - Estimated glomerular filtration rate (GFR) (ml/min) calculated by MDRD6 at baseline, SVR and 24 months after antiviral therapy $(p=0.02)$ 
density lipoprotein-cholesterol and high fasting glucose/ insulin-resistance. The post-LT metabolic syndrome prevalence is between 49 and $89 \%$ depending on the period after LT (17).

Increase in weight after LT is already known, with $58 \%$ overweight and respectively $21 \%$ obese recipients at 4 years post-LT (18). There is only one German study showing that, weight gain after DAA therapy and SVR occurred in $44 \%$ of patients after 48 weeks of follow-up, predominantly in patients younger than 60 years (19). Our study confirmed the increase in weight and BMI after SVR over the follow-up period. This was probably the cause of increase in BARD score, when all other fibrosis scores decreased with time passing from SVR. This is in agreement with our previous published article in LT HCV recipients (11), as well as in non-LT HCV population (20).

Although the majority of patients, will show evidence of fibrosis regression as time increases from HCV cure even in LT recipients (decrease of LS, APRI and FIB-4 scores, NAFLD scores) (11), some patients can progress. This may be related to the presence of nonalcoholic fatty liver disease, genetic or immunologic factors and concurrent alcohol use (21). Non-alcoholic fatty liver disease is correlated with metabolic syndrome which was estimated in our study by MetS score. In non-LT population, the severity of the metabolic syndrome is significantly combined with future coronary heart disease among patients with or without baseline type 2 diabetes. This is the first study to consider the evolution of MetS score in HCV LT recipients and there was no improvement during follow-up despite HCV cure. Metabolic syndrome serves as an important risk factor for atherosclerosis and cardiovascular disease, which are one of the principal causes of post-LT morbidity and mortality (22). Framingham risk score (FRS), already used in general population, to predict cardiovascular risk, could also be practical in the LT setting. Several studies $(23,24)$ have demonstrated that the FRS predicts with good accuracy the cumulative risk of developing post-LT cardiovascular events and renal function is an additional factor that independently predicts this risk. In the study by $\mathrm{Di}$ Maira et al (23), 9.3\%, 30.9\%, and $59.8 \%$ of HCVpositive patients and $21.5 \%, 22.4 \%$, and $56.1 \%$ of HCVnegative individuals $(p=N S)$, respectively, had a low, moderate, and high FRS at 1 year after LT (23). In our paper there was no recovery in the FRS score during the follow-up, but rather stabilization. To our knowledge, this is the first article assessing both MetS and FRS score in cured HCV LT recipients. The relationship between $\mathrm{HCV}$ infection, cardiovascular disorders, and renal impairment as previously described suggests that these variables play an essential role in determining an increased mortality risk $(8,25,26)$. However, in our study, eradication of HCV did not improve cardiovascular risk, contrary to reported data in some nontransplant populations $(27,28)$. In a LT setting, a previous paper (29) showed that shifting from cyclosporine to tacrolimus, led to stabilization of renal function, improvement of blood pressure profile and hyperlipidaemia as well as to a significant reduction in the Framingham risk score, independent of the aetiology of chronic liver disease. A more recent study (30) showed that even patients with mild chronic kidney disease (CKD) (stage 2) had a post-LT course marked by increased all-cause and cardiovascular mortality, highlighting the need for glomerular filtration rate preservation strategies as stated above.

Occurrence of renal dysfunction is frequent following LT and has a significant impact on morbidity and mortality. The degree of renal function impairment is related to an elevated risk of mortality, cardiovascular events, and prolonged hospital stay in liver transplant population (31). Over $50 \%$ of LT recipients have stage 3 CKD by 2 years post-LT and up to $20 \%$ progress to $\geq$ stage 4 within 5 years $(31,32)$. In the article by Watt et al, kidney failure was the cause of death for $10 \%$ of patients more than 5 years post-LT and associated CKD deaths were seen to progressively increase over follow-up (33).

Multiple factors contribute to CDK risk in LT such as older patient age, high blood pressure, diabetes mellitus, HCV, acute renal damage pre-transplantation and peri-transplantation, nephrotoxic effects of $\mathrm{CNIs}$, as well as sofosbuvir $(33,34)$.

Our results were rather contradictory to those from the previous 2 studies performed in HCV LT recipients; in our study, renal function impaired in $19.1 \%$ of patients with SVR. In the study of Beig et al. (10) there was a significant escalation in eGFR but still remaining within the same CKD stage 2 (eGFR increased from $64.9 \pm 20 \mathrm{~mL} /$ minutes prior to therapy to $69.6 \pm 20$ $\mathrm{mL} /$ minutes at 24 weeks after SVR). In the paper by Satapathy et al. (9), renal dysfunction still occurred, but patients with SVR had a less abrupt slope in eGFR decrease $(-0.60 \mathrm{~mL} /$ minute $/ 1.73 \mathrm{~m} 2 /$ year; $p=0.19)$ than recipients without SVR $(-2.53 \mathrm{~mL} /$ minute $/ 1.73$ $\mathrm{m} 2$ /year; $\mathrm{p}=0.001$ ). In our cohort, majority of patients received the 3D-regimen compared to the study of Beig et al. where most of patients had a sofosbuvir-based regimen (10). Another major difference is that renal dysfunction was evaluated after 24 weeks in the New Zealand study in comparison to 24 months in our study. 
Differences between LT cohorts, regarding renal function evolution over time, may depend on various factors related to study population characteristics: age, diabetes mellitus, hypertension, type and level of immunosuppression and antiviral therapy.

Herrero et al. (35) showed the key elements accounting for excellent renal function in the long term: good renal function before transplantation, stable $\mathrm{CNI}$ levels, minimization of $\mathrm{CNI}$ levels in patients with damaged renal function. One important variable in our study was the variation of the immunosuppression during co-administration of the 3D regimens, with higher trough levels at the beginning of antiviral therapy despite adjusting the $\mathrm{CNI}$ according to recommendations. A drawback of our study is the lack of a control cohort of non-HCV recipients followed up during the same period for prevalence and progression of CKD after LT in order to establish if renal impairment is immunosuppression or HCV-related. It should be mentioned that one third of our patients had already advanced liver disease due to recurrent HCV and the median time since LT to DAA initiation was over 2 years. The beneficial effect of SVR after LT on kidney and metabolic factors may be studied nowadays when antiviral therapy is initiated after the first 3 months following LT or to follow patients that are transplanted for complications of cirrhosis despite HCV cure.

Another finding of our study was the increase in cholesterol and glucose serum levels after SVR. In the era of DAA, elevated levels of cholesterol and LDLcholesterol following the clearance of HCV has been reported $(8,36)$. An increase in serum cholesterol could result in progression of atherosclerotic lesions; hence studies on the long-term dynamics of lipid profile following SVR and the effects on clinical outcomes, especially cardiovascular events, must be conducted in both LT and non-LT individuals. A meta-analysis (37) demonstrated, in non-LT patients, that SVR reduced the risk of insulin resistance at follow-up and had a significant protective effect against the incidence of new-onset type 2 diabetes. The influence of immunosuppression, weight increase and fatty liver disease/ advanced liver disease before DAA could not be assessed and may be a cause of increased glucose serum levels, and consequently increased insulin resistance, in our cohort. However, our results showing no beneficial effect of HCV eradication on glucose levels two years after SVR are in line with the review by Ribaldone et al (38). The authors concluded that there is no improvement in diabetes mellitus in patients with advanced fibrosis or with long-term diabetes mellitus history, suggesting that there is a point of no return for the modifications in glucose metabolism and, conversely, diabetes mellitus clinical outcome.

\section{CONCLUSION}

In summary, LT recipients achieving SVR in the DAA era exhibited a significant improvement of the liver graft function, decreased fibrosis scores, but without amelioration of metabolic/ cardiovascular factors and even worsening of renal function. In clinical practice, the transplant hepatologist should pay attention to cured HCV recipient with concomitant metabolic disorders and adopt life-style changes, body weight reduction, control of diabetes mellitus and modify immunosuppression according to these complications.

Conflicts of interest - none for all authors.

\section{Ethical approval}

The study protocol conformed to the ethical guidelines of the 1975 Declaration of Helsinki and was approved by the Institutional Review Board of Fundeni Clinical Institute. Because it is an observational study, performed with a retrospective design using a database and medical records, informed consent was waived by the board. However, all patients signed an informed consent in written form before inclusion on the waiting list for LT according to our Institute protocol, as well as before initiation, monitoring and follow-up during and after antiviral therapy. The study was conducted in concordance with the principles of the Declaration of Helsinki.

\section{REFERENCES}

1. Jain A, Reyes J, Kashyap R, Doson SF, Demetris AJ, Ruppert K, et al. Long-term survival after liver transplantation in 4000 consecutive patients at a single center. Ann Surg 2000;232:490-500.

2. Schoening WN, Buescher N, Rademacher S, Andreou A, Kuehn S, Neuhaus R, et al. Twenty-year longitudinal follow-up after orthotopic liver transplantation: a single-center experience of 313 consecutive cases. Am J Transplant 2013;13:2384-2394.

3. Duffy JP, Kao K, Ko CY, Farmer DG, McDiarmid SV, Hing JC, et al. Long-term patient outcome and quality of life after liver transplantation. Analysis of 20-year survivors. Ann Surg 2010;252:652-661.

4. Rubín A, Sánchez-Montes C, Aguilera V, Juan FS, Ferrer I, Moya A, et al. Long-term outcome of 'long-term liver transplant survivors'. Transpl Int. 2013;26:740-750.

5. Dopazo C, Bilbao I, Castells LL, Sapisochin G, Moreiras C, CamposVarela I, et al. Analysis of adult 20-year survivors after liver transplantation. Hepatol Int. 2015;9:461-470.

6. Ueda $Y$, Uemoto $S$. Interferon-Free Therapy for Hepatitis $C$ in Liver Transplant Recipients. Transplantation. 2016;100:54-60.

7. Wei L, Huang YH. Long-term outcomes in patients with chronic hepatitis $\mathrm{C}$ in the current era of direct-acting antiviral agents. Expert Rev Anti Infect Ther. 2019;17:311-325. 
8. Wang CC, Cheng PN, Kao JH. Systematic review: chronic vira hepatitis and metabolic derangement.Aliment Pharmacol Ther 2020;51:216-230.

9. Satapathy SK, Joglekar K, Molnar MZ, Ali B, Gonzalez HC, Vanatta $\mathrm{JM}$, et al. Achieving Sustained Virological Response in Liver Transplant Recipients With Hepatitis C Decreases Risk of Decline in Renal Function. Liver Transpl. 2018;24:1040-1049.

10. Beig J, Orr D, Harrison B, Gane E. Hepatitis C Virus Eradication with New Interferon-Free Treatment Improves Metabolic Profile in Hepatitis C Virus-Related Liver Transplant Recipients. Liver Transpl. 2018;24:1031-1039.

11. lacob S, Cerban R, Pietrareanu C, Ester C, lacob R, Gheorghe $C$ et al. $100 \%$ sustained virological response and fibrosis improvement in real-life use of direct acting antivirals in genotype-1b recurrent hepatitis $\mathrm{C}$ following liver transplantation. J Gastrointestin Liver Dis. 2018:27:139-144.

12. Lee AM, Gurka MJ, DeBoer MD. A metabolic syndrome severity score to estimate risk in adolescents and adults: current evidence and future potential. Expert Rev Cardiovasc Ther. 2016;14:411-413.

13. D'Agostino R, Vasan R, Pencina M, Wolf P, Cobain M, Massaro J, Kannel W. General Cardiovascular Risk Profile for Use in Primary Care: The Framingham Heart Study. Circulation 2008; 117: 743-753.

14. Grundy SM, Cleeman JI, Daniels SR, Donato KA, Eckel RH, Franklin $\mathrm{BA}$, et al. Diagnosis and management of the metabolic syndrome:an American Heart Association/National Heart, Lung and Blood Institutescientific statement. Circulation 2005; 112: 2735-2752.

15. Mauro E, Crespo G, Montironi C, Londoño MC, Hernández-Gea V, Ruiz $\mathrm{P}$, et al. Portal pressure and liver stiffness measurements in the prediction of fibrosis regression after SVR in recurrent hepatitis C. Hepatology 2018;67:1683-1694.

16. Berenguer M, Palau A, Aguilera V, Rayón JM, Juan FS, Prieto M. Clinical benefits of antiviral therapy in patients with recurrent hepatitis C following liver transplantation. Am J Transplant 2008; 8:679-687.

17. Vida Perez L, Montero Alvarez JL, Poyato Gonzalez A, Briceño Delgado J, Costan Rodero G, Fraga Rivas E, et al. Prevalence and Predictors of Metabolic Syndrome After Liver Transplantation. Transplant Proc. 2016;48:2519-2524.

18. Anastácio LR, Ferreira LG, de Sena Ribeiro H, Lima AS, Vilela EG, Toulson Davisson Correia MI. Body composition and over-weight of liver transplant recipients. Transplantation 2011;92:947-951.

19. Schlevogt B, Deterding K, Port K, Siederdissen CHZ, Sollik L, Kirschner J, et al. Interferon-free cure of chronic Hepatitis $\mathrm{C}$ is associated with weight gain during long-term follow-up. Z Gastroenterol. 2017;55:848-856.

20. Sporea I, Lupusoru R, Mare R, Popescu A, Gheorghe L, lacob S, Sirli R. Dynamics of liver stiffness values by means of transient elastography in patients with HCV liver cirrhosis undergoing interferon free treatment. J Gastrointestin Liver Dis. 2017;26:145-150.

21. Terrault N. Care of Patients Following Cure of Hepatitis C Virus Infection. Gastroenterol Hepatol (NY). 2018:14:629-634.

22. Watt KD, Charlton MR. Metabolic syndrome and liver transplantation: A review and guide to management. J Hepatol 2010;53 199-206.

23. Di Maira T, Rubin A, Puchades L, Aguilera V, Vinaixa C, Garcia M, et al. Framingham score, renal dysfunction, and cardiovascular risk in liver transplant patients. Liver Transpl. 2015;21:812-822.
24. Mansell H, Worobetz LJ, Sylwestrowicz T, Shoker AS. A retrospective study of the Framingham cardiovascular risk scores in a liver transplant population. Transplant Proc. 2013;45:308-314.

25. Kawaguchi T, Yoshida T, Harada M, Hisamoto T, Nagao Y, Ide T, et al. Hepatitis $C$ virus down-regulates insulin receptor substrates 1 and 2 through up-regulation of suppressor of cytokine signaling $3 . \mathrm{Am} \mathrm{J}$ Pathol 2004:165:1499-1508.

26. Vanni E, Abate ML, Gentilcore E, Hickman I, Gambino R, Cassader $M$, et al. Sites and mechanisms of insulin resistance in nonobese, nondiabetic patients with chronic hepatitis C. Hepatology 2009; 50:697-706.

27. Polo ML, Laufer N. Extrahepatic manifestations of HCV: the role of direct acting antivirals. Expert Rev Anti Infect Ther. 2017;15: 737-746.

28. Lledó G, Benítez-Gutiérrez L, Arias A, Requena S, Cuervas-Mons V, de Mendoza C. Benefits of hepatitis $C$ cure with antivirals: why test and treat? Future Microbiol. 2019;14:425-435.

29. Beckebaum S, Klein C, Varghese J, Sotiropoulos GC, Saner F, $\mathrm{Schmitz} \mathrm{K}$ et al. Renal function and cardiovascular risk profile after conversion from ciclosporin to tacrolimus: prospective study in 80 liver transplant recipients. Aliment Pharmacol Ther. 2009;30: 834-842.

30. VanWagner LB, Montag S, Zhao L, Allen N, Lloyd-Jones D, Das A, et al.Cardiovascular Disease Outcomes Related to Early Stage Renal Impairment Following Liver Transplantation. Transplantation. 2018; 102:1096-1107.

31. Ojo AO, Held PJ, Port FK, Wolfe RA, Leichtman AB, Young EW, et al. Chronic renal failure after transplantation of a nonrenal organ. N Engl J Med 2003:349:931-940.

32. Guitard J, Ribes D, Kamar N, Muscari F, Cointault O, Lavayssière L, et al. Predictive factors for chronic renal failure one year after orthotopic liver transplantation. Ren Fail. 2006; 28:419-425.

33. Watt KD, Pedersen RA, Kremers WK, Heimbach JK, Charlton MR. Evolution of causes and risk factors for mortality post-liver transplant: results of the NIDDK long-term follow-up study. Am J Transplant 2010;10:1420-1427.

34. Dharancy S, Coilly A, Fougerou-Leurent C, Duvoux C, Kamar N, Leroy $\mathrm{V}$, et al. Direct-acting antiviral agent-based regimen for HCV recurrence after combined liver-kidney transplantation: Results from the ANRS C023 CUPILT study. Am J Transplant. 2017; 17:2869-2878.

35. Herrero JI, Cuervas-Mons V, Gomez-Bravo MA, Fabregat J, Otero A, Bilbao I, et al. Prevalence and progression of chronic kidney disease after a liver transplant: a prospective, real-life, observational, two-year multicenter study. Rev Esp Enferm Dig 2018; 110:538-543.

36. Bassendine MF, Nielsen SU, Bridge SH, Felmlee DJ, Sheridan DA, Packard CJ, Neely RD. Hepatitis C virus and atherosclerosis: A legacy after virologic cure? Clin Res Hepatol Gastroenterol. 2017; 41:25-30.

37. Cacoub P, Desbois AC, Comarmond C, Saadoun D. Impact of sustained virological response on the extrahepatic manifestations of chronic hepatitis C: a meta-analysis. Gut. 2018;67:2025-2034.

38. Ribaldone DG, Sacco M, Saracco GM. The Effect of Viral Clearance Achieved by Direct-Acting Antiviral Agents on Hepatitis C Virus Positive Patients with Type 2 Diabetes Mellitus: A Word of Caution after the Initial Enthusiasm. J Clin Med 2020:9:563. 